\title{
Duration discrimination in the range of milliseconds and seconds in children with ADHD and their unaffected siblings
} \author{
A. Rothenberger ${ }^{1}$ and T. Rammsayer ${ }^{3}$ \\ ${ }^{1}$ Child and Adolescent Psychiatry, University of Goettingen, Germany \\ ${ }^{2}$ Child and Adolescent Psychiatry, Central Institute of Mental Health, Mannheim, Germany \\ ${ }^{3}$ Department of Psychology, University of Bern, Switzerland
}

S. Himpel ${ }^{1 *}$, T. Banaschewski ${ }^{2}$, A. Grüttner ${ }^{1}$, A. Becker ${ }^{1}$, A. Heise ${ }^{1}$, H. Uebel ${ }^{1}$, B. Albrecht ${ }^{1}$,

\begin{abstract}
Background. Detecting genetic factors involved in attention deficit hyperactivity disorder (ADHD) is complicated because of their small effect sizes and complex interactions. The endophenotype approach eases this by coming closer to the relevant genes. Different aspects of temporal information processing are known to be affected in ADHD. Thus, some of these aspects could represent candidate endophenotypes for ADHD.
\end{abstract}

Method. Fifty-four sib-pairs with at least one child with ADHD and 40 control children aged 6-18 years were recruited and asked to perform two duration discrimination tasks, one with a base duration of $50 \mathrm{~ms}$ on automatic timing and one with a base duration of $1000 \mathrm{~ms}$ on cognitively controlled timing.

Results. Whereas children with ADHD, but not their unaffected siblings, were impaired in discrimination of longer intervals, both groups were impaired in discriminating brief intervals. Furthermore, a significant within-family correlation was found for discrimination of brief intervals. Task performances of subjects of the control group correlated with individual levels of hyperactivity/impulsivity for discrimination of brief intervals, but not of longer intervals.

Conclusions. Cognitively controlled and also automatic processes of temporal information processing are impaired in children with ADHD. Discrimination of longer intervals appears as a typical 'disease marker' whereas discrimination of brief intervals shows up as a 'vulnerability marker'. Discrimination of brief intervals was found to be familial and linked to levels of hyperactivity/impulsivity. Taken together, discrimination of brief intervals represents a candidate endophenotype of ADHD.

Received 11 September 2008; Accepted 14 January 2009; First published online 6 March 2009

Key words: Attention deficit hyperactivity disorder, endophenotypes, duration discrimination task, automatic timing, cognitively controlled timing.

\section{Introduction}

Attention deficit hyperactivity disorder (ADHD) is a highly heritable disorder (Faraone et al. 2005). Family studies consistently reveal an estimated sibling risk ratio of three- to sixfold (Faraone \& Doyle, 2000). However, underlying genetic factors are expected to be multiple, have small effect sizes when considered individually, and interact with each other and with environmental factors (Asherson, 2004). One approach to overcome these difficulties is the search for endophenotypes. The endophenotype concept represents a

* Address for correspondence: Dr S. Himpel, Child and Adolescent Psychiatry, University of Goettingen, von-Siebold-Str. 5, 37075

Goettingen, Germany.

(Email: shimpel@gwdg.de) strategy to analyse the biological systems beyond the obvious disorder (de Geus, 2002; Gottesman \& Gould, 2003). Endophenotypes are assumed to be influenced by one or more of the same genes as the disorder but to be regulated more directly by these genes. They should thus be less genetically complex than the disorder they underlie. Endophenotypes should have good psychometric properties and be stable over time (be reliably measurable); be associated with the disorder in the general population and co-segregate with the disorder in families; and be heritable (or at least familial) and show increased expression in unaffected relatives of affected subjects.

Several studies have shown deficits in temporal information processing in children with ADHD in a wide variety of tasks, such as time reproduction, verbal time estimation, anticipation, finger tapping and duration 
discrimination tasks (for a review see Toplak et al. 2006). Although the results in time reproduction, duration discrimination and finger tapping tasks are fairly consistent in showing deficits in task performance for children and adolescents with ADHD, less consistent results have been obtained in verbal estimation and anticipation tasks. However, most results are available on time reproduction tasks. Most recently, Rommelse et al. (2007) showed that children with ADHD, and their non-affected siblings, performed less well in a time reproduction task with intervals between 4 and $20 \mathrm{~s}$ compared to normal controls. On the basis of these results, they proposed time reproduction as a candidate endophenotype for ADHD.

In the field of human temporal information processing, the classical single-clock models hypothesize a general timing mechanism underlying temporal information processing across a wide range of durations (Matell \& Meck, 2000). However, there are many results from psychophysical (Rammsayer \& Lima, 1991; Karmarkar \& Buonomano, 2007) and pharmacopsychological studies (Rammsayer, 1992a, 1993, 1997, 1999, 2006; Rammsayer et al. 2001) that cannot be explained by a single timing mechanism for the whole range of time. These findings support the idea of two (or even more) different timing mechanisms. Thus, a two-process model with an automatic mechanism for timing in the range of milliseconds and a cognitively controlled mechanism for timing in the second range has been proposed, with transition from one timing mechanisms to the other somewhere between 200 and 800 ms (Rammsayer \& Lima, 1991; Ivry, 1996; Lewis \& Miall, 2003a, b, 2006; Karmarkar \& Buonomano, 2007). This notion is further supported by brain lesion and neuroimaging studies; brief intervals in the range of milliseconds seem to be processed in primary sensorimotor and premotor circuits whereas longer intervals tend to recruit right hemispheric prefrontal and parietal cortices (Mangels et al. 1998; Rao et al. 2001; Lewis \& Miall, 2003a, b).

In the present study, temporal information processing was analysed in children with ADHD and their unaffected siblings to search for candidate endophenotypes for ADHD. The study also focused on the differentiation between automatic and cognitively controlled timing by applying two versions of a duration discrimination task, one with base durations of $50 \mathrm{~ms}$ and one with base durations of $1000 \mathrm{~ms}$.

\section{Method \\ Subjects}

Siblings were recruited from families participating in the Goettingen subsample of the International Multi- center ADHD Genes (IMAGE) study (Brookes et al. 2006). Families were included if at least one child suffered from ADHD and had at least one available sibling, regardless of whether the sibling was also affected or not. The complete study had medical/ ethical approval from the National Institute of Mental Health (NIMH) confirmed by the local ethical review board.

Diagnosis of ADHD was made using the standard procedures of the IMAGE project as described in detail elsewhere (Brookes et al. 2006). In brief, children were screened for ADHD by applying Conners' rating scales (parent and teacher Conners' long-version rating scales) (Conners, 1996) and Strengths and Difficulties Questionnaires (parent and teacher SDQ-D; Goodman, 1997; Woerner et al. 2004). If ADHD was indicated by these rating scales, it was confirmed clinically by a semi-structured standardized clinical interview, the Parental Account of Children's Symptoms (PACS; Taylor, 1991). PACS interviews were conducted for each child separately by well-trained medical clinicians. Children not being considered as suffering from ADHD by Conners' and SDQ scales were not interviewed with PACS. Each pair of siblings consisted of a child with ADHD and his/her available sibling with the smallest age difference.

A normal control group was selected from primary and high schools. Control children were included if they had non-clinical scores on Conners' $\mathrm{N}$ subscales of both parent and teacher Conners' long-version rating scales ( $T$ score $\leqslant 62$ ) and had no known psychiatric disorder.

All children were 6- to 18-year-old European Caucasians with normal hearing. Exclusion criteria for all groups were $\mathrm{IQ}<70$ or a diagnosis of autism spectrum disorder, bipolar or schizophrenic psychosis, brain disorder, epilepsy, or known genetic disorders that might mimic ADHD.

By this procedure, 62 pairs of siblings were recruited with 10 pairs concordant for ADHD and 52 pairs discordant for ADHD. Thus, 72 children with ADHD and 52 unaffected siblings were recruited for the initial sample. In addition, 44 normal control children were included.

\section{Intelligence}

IQ was estimated by four subtests of the Wechsler Intelligence Scale for Children - Third Edition (WISCIII) or the Wechsler Adult Intelligence Scale - Third Edition (WAIS-III), depending on the child's age (Wechsler, 2000, 2002). The vocabulary, similarities, picture completion and block design subtests were used to obtain an estimate of the child's IQ (Sattler, 1992; Groth-Marnat, 1997). 


\section{Duration discrimination tasks}

Duration discrimination tasks were carried out as described previously (Rammsayer et al. 2001). In brief, the performances on duration discrimination were assessed by presenting pairs of auditory stimuli, a standard interval and a variable comparison interval, and asking the subjects to decide which of the two intervals was longer. The instructions to the participants emphasized accuracy; there was no requirement to respond quickly. After each response, visual feedback $\left({ }^{\prime}+{ }^{\prime}=\right.$ correct or ${ }^{\prime}-'=$ false $)$ was displayed on the monitor screen. An experimental session consisted of one block with a standard interval of $1000 \mathrm{~ms}$ and one block with a standard interval of $50 \mathrm{~ms}$ each. Each block consisted of 32 trails. The order of blocks and the presentation order of standard and comparison intervals within each block were counterbalanced across participants. For assessing performance on temporal discrimination, an adaptive psychophysical procedure was used to determine the $75 \%$ difference threshold for the 50-ms and 1000-ms standard intervals respectively (Kaernbach, 1991; Rammsayer, 1992b). The 75\% difference threshold represents the difference in duration between the standard and comparison intervals required to produce $75 \%$ correct responses. As a measure of performance, threshold values were computed based on mid-run estimates for the last 20 trials within each block (Wetherill et al. 1966). The individual threshold represents a psychophysical measure of performance indicating the just noticeable difference between standard and comparison intervals. Thus, better performances are indicated by smaller thresholds.

\section{Data analyses}

Initially, data were checked for completeness and children with incomplete data and if available, from the corresponding sibling were excluded from further analysis. In addition, data were checked for outliers. Complete data sets were excluded if performance in one or more tasks differed from the mean of the respective group for more than two standard deviations.

Phenotypic analyses were conducted using SPSS version 15.0 (SPSS Inc., Chicago, IL, USA). All dependent variables were nearly normally distributed (Kolmogoroff-Smirnov $Z=0.659-1.025, p=0.24-0.78$ ). The level of statistical significance was set at 0.05 for all analyses. Univariate analysis of variance (ANOVA) was used to examine group effects on measured discrimination thresholds for each task separately. Group was set as a fixed factor and family as a random effect to account for family clustering. Age was used as a covariate because a significant effect on task performance was found for both conditions $\left[F(1,113)=11.24, p=0.001, \eta^{2}=0.091\right.$, and $F(1,103)=$ $31.29, p<0.001, \eta^{2}=0.23$ for time estimation and time perception respectively]. In addition, IQ and gender were initially introduced as covariates into the model, but because no significant effects on dependent variables were found, they were finally removed. Main effects were examined by means of pair-wise comparison with Sidak's correction for multiple comparisons. Effect sizes were estimated by calculating Cohen's $d$, with the difference of estimated means divided by pooled standard deviations (Cohen, 1998). Within-family correlations of task performance were examined by calculating the partial correlation coefficients of thresholds between siblings controlling for age of both siblings (sib-pair correlations). To investigate correlations between task performances and individual ADHD symptom levels, partial correlation coefficients between thresholds and Conners' scores of the L (DSM-IV inattentive), M (DSM-IV hyperactive/ impulsive) and N (DSM-IV total) subscales (averaged across parent and teacher ratings), controlling for age, were calculated. Because of a high correlation between parent and teacher ratings $(r=0.72-0.79, p<0.001)$, parent and teacher ratings of these Conners' subscales were averaged.

\section{Results}

Eight pairs of siblings and four control children were excluded from the initial sample because of incomplete or outlying task performance. Thus, the final sample included 54 pairs of siblings with 63 affected and 45 unaffected children and 40 control children. Table 1 presents the main group characteristics. There was no significant group difference for age and IQ, but there was a significant group difference for gender ratio. Group had a significant influence on all three Conners' ADHD scores with the ADHD group differing significantly from the group of unaffected siblings and the group of control children. There was no significant difference between the groups of unaffected siblings and control children.

\section{0-ms standard interval}

For the duration discrimination task with a base duration of $50 \mathrm{~ms}$, the mean $75 \%$ difference threshold ( \pm S.D.) was $20.8 \pm 8.0 \mathrm{~ms}$ for the ADHD group, $19.7 \pm$ $8.5 \mathrm{~ms}$ for the unaffected sibling group, and 16.6 \pm $6.3 \mathrm{~ms}$ for the control group. A significant effect of group was found $\left[F(2,108)=4.01, p=0.021, \eta^{2}=0.07\right]$. Pair-wise comparisons revealed that children with ADHD and their unaffected siblings performed significantly worse than controls $(p=0.002, d=0.84$ and 
Table 1. Sample characteristics

\begin{tabular}{|c|c|c|c|c|c|c|c|c|c|}
\hline & \multicolumn{2}{|c|}{$\begin{array}{l}\text { ADHD group } \\
(n=63)\end{array}$} & \multicolumn{2}{|c|}{$\begin{array}{l}\text { Unaffected group } \\
(n=45)\end{array}$} & \multicolumn{2}{|c|}{$\begin{array}{l}\text { Control group } \\
(n=40)\end{array}$} & \multirow[b]{2}{*}{$F(2,145)$} & \multirow[b]{2}{*}{$p$} & \multirow[b]{2}{*}{$\eta^{2}$} \\
\hline & Mean & S.D. & Mean & S.D. & Mean & S.D. & & & \\
\hline Age (years ;months) & $11 ; 3$ & $2 ; 3$ & $11 ; 7$ & $3 ; 1$ & $11 ; 4$ & $2 ; 2$ & $0.30^{\mathrm{a}}$ & 0.74 & 0.004 \\
\hline IQ & 102.7 & 11.4 & 105.2 & 12.4 & 107.3 & 13.1 & $1.88^{\mathrm{a}}$ & 0.16 & 0.03 \\
\hline Gender ( $\%$ male $)$ & 92.1 & & 46.7 & & 62.5 & & $27.5^{\mathrm{b}}$ & $<0.001$ & \\
\hline \multicolumn{10}{|l|}{ Averaged Conners' ADHD scores } \\
\hline DSM-IV : inattentive & 68.2 & 7.2 & 50.7 & 6.8 & 49.9 & 8.0 & $108.9^{\mathrm{a}}$ & $<0.001^{\mathrm{c}}$ & 0.60 \\
\hline DSM-IV : hyperactive-impulsive & 73.4 & 7.5 & 48.2 & 4.2 & 49.2 & 7.0 & $259.7^{\mathrm{a}}$ & $<0.001^{\mathrm{c}}$ & 0.78 \\
\hline DSM-IV : total & 72.4 & 7.3 & 49.7 & 5.3 & 49.7 & 7.4 & $203.8^{\mathrm{a}}$ & $<0.001^{\mathrm{c}}$ & 0.74 \\
\hline
\end{tabular}

ADHD, Attention deficit hyperactivity disorder; S.D., standard deviation.

${ }^{\text {a }}$ One-way ANOVA.

${ }^{\mathrm{b}} \chi^{2}$ test.

${ }^{\mathrm{c}}$ Post-hoc pair-wise Sidak-corrected $t$ tests : ADHD group differs significantly from control group $(p<0.05)$ and from unaffected group $(p<0.05)$.

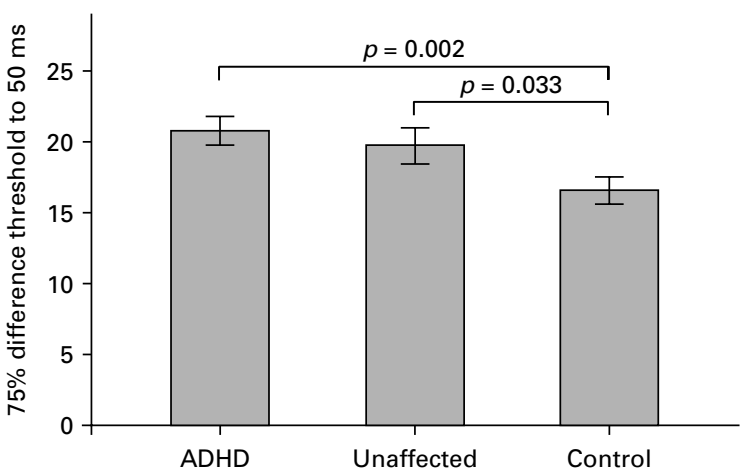

Fig. 1. Performance on discrimination of brief intervals as indicated by the $75 \%$ difference threshold in relation to a 50-ms standard interval for attention deficit hyperactivity disorder-affected children (ADHD), unaffected siblings (unaffected), and normal control children (control) (mean \pm S.E.M., $p$ of Sidak-corrected pairwise comparisons).

$p=0.033, d=0.61$ respectively) (see Fig. 1 ). The difference between children with ADHD and their unaffected siblings was not significant ( $p=0.55, d=0.24$ ).

The group effect remained significant after introducing gender as a covariate $[F(2,109)=3.84, p=0.024$, $\left.\eta^{2}=0.07\right]$. In addition, there was no significant difference in task performance between boys and girls for all three groups according to Mann-Whitney $U$ tests $(Z=0.09, p=0.93 ; Z=0.33, p=0.74 ;$ and $Z=0.66, p=$ 0.51 for ADHD children, unaffected siblings, and control children respectively).

Furthermore, siblings within families resembled each other in their task performance as indicated by the sib-pair correlation $(r=0.39, p=0.004)$. Similar results were obtained after excluding pairs of siblings with both being affected $(r=0.37, p=0.015)$.
In the control group, phenotypic correlation analyses between individual $75 \%$ difference thresholds and corresponding DSM-IV Conners' $\mathrm{M}$ and $\mathrm{N}$ scores showed a significant correlation between performance and levels of hyperactivity/impulsivity (M scores) and total ADHD symptoms ( $\mathrm{N}$ scores) $(r=0.32$, $p=0.046$ and $r=0.32, p=0.047$ respectively). The correlation between performance and individual levels of inattention (L scores) failed to reach the $5 \%$ level of statistical significance $(r=0.27, p=0.092)$.

\section{0-ms standard interval}

For the duration discrimination task with a base duration of $1000 \mathrm{~ms}$, the mean $75 \%$ difference threshold ( \pm s.D.) was $344.0 \pm 174.3 \mathrm{~ms}$ for the ADHD group, $252.5 \pm 102.5 \mathrm{~ms}$ for the unaffected sibling group, and $215.1 \pm 83.6 \mathrm{~ms}$ for the control group. A significant effect of group was found $[F(2,87)=11.73, p<0.001$, $\left.\eta^{2}=0.21\right]$. Pair-wise comparisons revealed that children with ADHD performed significantly worse than their unaffected siblings $(p=0.002, d=0.81)$ and significantly worse than controls $(p<0.001, d=1.13)$ (see Fig. 2). The difference between unaffected siblings and controls did not reach significance ( $p=0.35, d=0.34$ ).

The group effect stayed significant after introducing gender as a covariate $\left[F(2,90)=9.24, p<0.001, \eta^{2}=\right.$ $0.17]$. In addition, there was no significant difference in task performance between boys and girls for all three groups according to Mann-Whitney $U$ tests $(Z=1.22$, $p=0.22 ; Z=1.12, p=0.27$; and $Z=0.13, p=0.9$ for ADHD children, unaffected siblings, and control children respectively). For the comparison of task performance of siblings, the sib-pair correlation did not reach significance $(r=0.25, p=0.069)$. For the control 


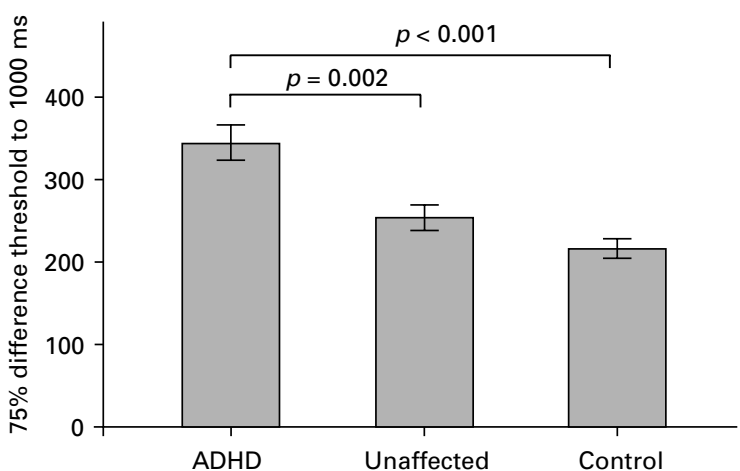

Fig. 2. Performance on discrimination of longer intervals as indicated by the $75 \%$ difference threshold in relation to a 1000-ms standard interval of attention deficit hyperactivity disorder-affected children (ADHD), unaffected siblings (unaffected), and normal control children (control) (mean \pm S.E.M., $p$ of Sidak-corrected pairwise comparisons).

group, phenotypic correlations between individual $75 \%$ difference thresholds and corresponding DSM-IV Conners' scores (L, M and N) were $r=0.24, r=-0.20$ and $r=0.08$ respectively, and all failed to reach the $5 \%$ level of statistical significance. Thus, there was no indication for a functional relationship between performance and levels of ADHD symptom dimensions.

\section{Discussion}

The present study showed that performances on discrimination of extremely brief intervals in the range of several tens of milliseconds and of longer intervals in the 1-s range are clearly impaired in children with ADHD compared to normal controls, indicating deficits in automatic and also in cognitively controlled temporal information processing. By contrast, unaffected siblings of children with ADHD differ from normal controls in their performance to discriminate brief intervals in the range of milliseconds, but not to discriminate longer intervals. This indicates that these siblings are impaired in automatic timing mechanisms although they do not suffer from ADHD and their Conners' ADHD scores do not differ from those of normal control children. On the basis of these results, impaired duration discrimination in the second range can be seen as a 'disease marker' whereas duration discrimination in the range of several tens of milliseconds fits the pattern of a 'vulnerability marker' that distinguishes between normal families and families with an increased risk for ADHD.

Rommelse et al. (2007) found that children with ADHD, and their unaffected siblings, were less precise in time reproduction of intervals in the range of 4 to $20 \mathrm{~s}$. Thus, time reproduction has been proposed as a candidate endophenotype for ADHD. At first glance, this is somewhat at variance with our findings of no endophenotypic pattern for duration discrimination of intervals in the 1-s range. In contrast to duration discrimination tasks, time reproduction tasks primarily involve aspects of time perception, time estimation and time production, and are also dependent on motor timing and coordination (Zakay, 1990). Rommelse et al. 2008a,b) also found endophenotypic patterns for motor timing and oculomotor control in families with ADHD. Thus, their findings of endophenotypic patterns in time reproduction might result from endophenotypic patterns in motor timing or oculomotor coordination. In general, temporal information processing is a complex neuropsychological function. Duration discrimination and time reproduction are thus not based on the same set of timing mechanisms and results cannot be compared directly.

The applied duration discrimination task with a 50ms standard interval and its differentiation from the 1000-ms version have been validated by various timing studies with healthy humans (Rammsayer, 1992a, 1994; Rammsayer et al. 1993; Rammsayer \& Ulrich, 2001; Rammsayer \& Brandler, 2004). Furthermore, pharmacopsychological and dual-task studies have frequently shown separability of the two task versions (Rammsayer, 1989, 1993, 1999, 2006; Rammsayer \& Vogel, 1992; Rammsayer et al. 2001). Thus, duration discrimination in the range of milliseconds is reliably measurable, a necessary condition for candidate endophenotypes.

For duration discrimination of brief intervals, the values obtained were correlated significantly to levels of total ADHD symptoms in the normal control group, especially to the hyperactive/impulsive symptom level. This provides, on a dimensional level, further evidence for the association between worse performance in duration discrimination in the range of milliseconds and ADHD phenotypology. Furthermore, high resemblances between siblings within families were observed, as apparent from significant positive sib-pair correlations. This indicates further familiality of duration discrimination of brief intervals. Thus, overall, discrimination of intervals in the range of several tens of milliseconds represents a candidate endophenotype for ADHD.

\section{Limitations}

The present study was based on a sibling design. Whereas twin designs produce the three estimates of heritability, shared environmental influences and child-specific environmental influences, sibling designs cannot distinguish between genetic influences and shared environmental influences. They are accordingly only able to differentiate between familiality 
and child-specific influences, with familiality reflecting the combination of genetic and shared environmental effects. Thus, the observed familiality of performances in duration discrimination of brief intervals does not necessarily prove heritability.

The sample consisted mainly of boys and the groups were not matched for gender (Table 1). However, a potential influence of gender was not found for task performance of girls and boys within each group for both long and short intervals.

\section{Acknowledgements}

Sample recruitment was supported by NIMH Grant R01MH062873 to the IMAGE project.

\section{Declaration of Interest}

None.

\section{References}

Asherson P (2004). Attention-deficit hyperactivity disorder in the post-genomic era. European Child and Adolescent Psychiatry 13 (Suppl. 1), 50-70.

Brookes K, Xu X, Chen W, Zhou K, Neale B, Lowe N, Anney R, Franke B, Gill M, Ebstein R, Buitelaar J, Sham P, Campbell D, Knight J, Andreou P, Altink M, Arnold R, Boer F, Buschgens C, Butler L, Christiansen H, Feldman L, Fleischman K, Fliers E, Howe-Forbes R, Goldfarb A, Heise A, Gabriels I, Korn-Lubetzki I, Johansson L, Marco R, Medad S, Minderaa R, Mulas F, Muller U, Mulligan A, Rabin K, Rommelse N, Sethna V, Sorohan J, Uebel H, Psychogiou L, Weeks A, Barrett R, Craig I, Banaschewski T, Sonuga-Barke E, Eisenberg J, Kuntsi J, Manor I, McGuffin P, Miranda A, Oades RD, Plomin R, Roeyers H, Rothenberger A, Sergeant J, Steinhausen HC, Taylor E, Thompson M, Faraone SV, Asherson P (2006). The analysis of 51 genes in DSM-IV combined type attention deficit hyperactivity disorder: association signals in DRD4, DAT1 and 16 other genes. Molecular Psychiatry 11, 934-953.

Cohen J (1998). Statistical Power Analysis for the Behavioral Sciences. Lawrence Earlbaum Associates: Hillsdale, NJ.

Conners K (1996). Rating Scales in ADHD. Duke University Medical Center: Durham, NC.

de Geus EJ (2002). Introducing genetic psychophysiology. Biological Psychology 61, 1-10.

Faraone SV, Doyle AE (2000). Genetic influences on attention deficit hyperactivity disorder. Current Psychiatry Reports 2, 143-146.

Faraone SV, Perlis RH, Doyle AE, Smoller JW, Goralnick JJ, Holmgren MA, Sklar P (2005). Molecular genetics of attention-deficit/hyperactivity disorder. Biological Psychiatry 57, 1313-1323.

Goodman R (1997). The Strengths and Difficulties Questionnaire: a research note. Journal of Child Psychology and Psychiatry and Allied Disciplines 38, 581-586.
Gottesman II, Gould TD (2003). The endophenotype concept in psychiatry: etymology and strategic intentions. American Journal of Psychiatry 160, 636-645.

Groth-Marnat G (1997). Handbook of Psychological Assessment. Wiley: New York.

Ivry RB (1996). The representation of temporal information in perception and motor control. Current Opinion in Neurobiology 6, 851-857.

Kaernbach C (1991). Simple adaptive testing with the weighted up-down method. Perception and Psychophysics 49, 227-229.

Karmarkar UR, Buonomano DV (2007). Timing in the absence of clocks: encoding time in neural network states. Neuron 53, 427-438.

Lewis PA, Miall RC (2003a). Distinct systems for automatic and cognitively controlled time measurement: evidence from neuroimaging. Current Opinion in Neurobiology 13, 250-255.

Lewis PA, Miall RC (2003b). Brain activation patterns during measurement of sub- and supra-second intervals. Neuropsychologia 41, 1583-1592.

Lewis PA, Miall RC (2006). Remembering the time: a continuous clock. Trends in Cognitive Science 10, 401-406.

Mangels JA, Ivry RB, Shimizu N (1998). Dissociable contributions of the prefrontal and neocerebellar cortex to time perception. Brain Research. Cognitive Brain Research 7, 15-39.

Matell MS, Meck WH (2000). Neuropsychological mechanisms of interval timing behavior. Bioessays 22, 94-103.

Rammsayer T (1989). Dopaminergic and serotoninergic influence on duration discrimination and vigilance. Pharmacopsychiatry 22 (Suppl. 1), 39-43.

Rammsayer T (1992a). Time perception as a useful tool for measuring drug effects unrelated to vigilance and sedation. Clinical Neuropharmacology 15 (Suppl. 1), 594A-595A.

Rammsayer T (1992b). An experimental comparison of the weighted up-down method and the transformed up-down method. Bulletin of the Psychonomic Society 30, 425-427.

Rammsayer T (1993). On dopaminergic modulation of temporal information processing. Biological Psychology 36, 209-222.

Rammsayer T (1994). Effects of practice and signal energy on duration discrimination of brief auditory intervals. Perception and Psychophysics 55, 454-464.

Rammsayer T (1997). Effects of body core temperature and brain dopamine activity on timing processes in humans. Biological Psychology 46, 169-192.

Rammsayer T (1999). Neuropharmacological evidence for different timing mechanisms in humans. Quarterly Journal of Experimental Psychology 52, 273-286.

Rammsayer T (2006). Effects of pharmacologically induced changes in NMDA receptor activity on human timing and sensorimotor performance. Brain Research 1073-1074, 407-416.

Rammsayer T, Brandler S (2004). Aspects of temporal information processing: a dimensional analysis. Psychological Research 69, 115-123.

Rammsayer T, Hennig J, Haag A, Lange N (2001). Effects of noradrenergic activity on temporal information processing 
in humans. Quarterly Journal of Experimental Psychology 54, 247-258.

Rammsayer T, Lima SD (1991). Duration discrimination of filled and empty auditory intervals: cognitive and perceptual factors. Perception and Psychophysics 50, 565-574.

Rammsayer T, Lima SD, Vogel WH (1993). Aging and temporal discrimination of brief auditory intervals. Psychological Research 55, 15-19.

Rammsayer T, Ulrich R (2001). Counting models of temporal discrimination. Psychonomic Bulletin and Review 8, 270-277.

Rammsayer T, Vogel WH (1992). Pharmacologic properties of the internal clock underlying time perception in humans. Neuropsychobiology 26, 71-80.

Rao SM, Mayer AR, Harrington DL (2001). The evolution of brain activation during temporal processing. Nature Neuroscience 4, 317-323.

Rommelse NN, Altink ME, Oosterlaan J, Beem L, Buschgens CJ, Buitelaar J, Sergeant JA (2008a). Speed, variability, and timing of motor output in ADHD: which measures are useful for endophenotypic research? Behavior Genetics 38, 121-132.

Rommelse NN, Oosterlaan J, Buitelaar J, Faraone SV, Sergeant JA (2007). Time reproduction in children with ADHD and their nonaffected siblings. Journal of the American Academy of Child and Adolescent Psychiatry 46, 582-590.
Rommelse NN, Van der Stigchel S, Witlox J, Geldof C, Deijen JB, Theeuwes J, Oosterlaan J, Sergeant JA (2008b). Deficits in visuo-spatial working memory, inhibition and oculomotor control in boys with ADHD and their non-affected brothers. Journal of Neural Transmission 115, 249-260.

Sattler JM (1992). Assessment of Children: WISC-III and WPPSI-R Supplement. Jerome M. Sattler: San Diego, CA.

Taylor E, Sandberg S, Thorley G, Giles S (1991). The Epidemiology of Childhood Hyperactivity. Oxford University Press: New York.

Wechsler D (2000). The Netherlands Transcription of WAIS-III. Technical Instructions. The Psychological Corporation: London.

Wechsler D (2002). WISC-III Handbook. The Psychological Corporation: London.

Wetherill GB, Chen H, Vasudeva RB (1966). Seqential estimation of quantal response curves: a new method of estimation. Biometrika 53, 439-454.

Woerner W, Becker A, Rothenberger A (2004). Normative data and scale properties of the German parent SDQ. European Child and Adolescent Psychiatry 13 (Suppl. 2), II3-II10.

Zakay D (1990). The evasive art of subjective time measurement: some methodological dilemmas. In Cognitive Models of Psychological Time (ed. R.A. Block), pp. 59-84. Lawrence Erlbaum Associates: Hillsdale, NJ. 